\title{
h-EXPONENTIAL CHANGE OF FINSLER METRIC
}

\author{
Manish Kumar Gupta* and Anil K. Gupta
}

\begin{abstract}
In this paper, we studied a Finsler space whose metric is given by an $h$ exponential change and obtain the Cartan connection coefficients for the change. We also find the necessary and sufficient condition for an $h$-exponential change of Finsler metric to be projective.
\end{abstract}

Keywords:Finsler space; $h$-exponential change; Projective change

\section{Introduction}

Let $F^{n}=\left(M^{n}, L\right)$ be an $n$-dimensional Finsler space equipped with the fundamental function $L(x, y)$. The metric tensor, angular metric tensor and Cartan tensor are defined by $g_{i j}=\frac{1}{2} \dot{\partial}_{i} \dot{\partial}_{j} L^{2}, h_{i j}=g_{i j}-l_{i} l_{j}$ and $C_{i j k}=\frac{1}{2} \dot{\partial}_{i} g_{j k}$ respectively, where $\dot{\partial}_{k}=\frac{\partial}{\partial y^{k}}$. The Cartan connection is given by $C \Gamma=\left(F_{j k}^{i}, N_{k}^{i}, C_{j k}^{i}\right)$. The $h$ - and v-covariant derivatives $X_{i \mid j}$ and $\left.X_{i}\right|_{j}$ of a covariant vector field $X_{i}$ are defined by $[10,14]$

$$
\begin{gathered}
X_{i \mid j}=\partial_{j} X_{i}-N_{j}^{r} \dot{\partial}_{r} X_{i}-X_{r} F_{i j}^{r}, \\
\left.X_{i}\right|_{j}=\dot{\partial}_{j} X_{i}-X_{r} C_{i j}^{r},
\end{gathered}
$$

where $\partial_{k}=\frac{\partial}{\partial x^{k}}$.

In 2012, H. S. Shukla et.al.[15] considered a Finsler space $\bar{F}^{n}=\left(M^{n}, \bar{L}\right)$, whose Fundamental metric function is an exponential change of Finsler metric function given by

$$
\bar{L}=L e^{\frac{\beta}{L}},
$$

where $\beta=b_{i}(x) y^{i}$ is 1 -form on manifold $M^{n}$.

Received February 02, 2016; accepted April 28, 2016

2010 Mathematics Subject Classification. 53B40

${ }^{*}$ First author is supported by UGC, Government of India. 
H. Izumi [6] introduced the concept of an $h$-vector $b_{i}(x, y)$ which is $v$-covariant constant with respect to the Cartan connection and satisfies $L C_{i j}^{h} b_{h}=\rho h_{i j}$, where $\rho$ is a non-zero scalar function and $C_{j k}^{i}$ are components of Cartan tensor. Thus if $b_{i}$ is an $h$-vector then

$$
\text { (i) }\left.b_{i}\right|_{k}=0, \quad \text { (ii) } L C_{i j}^{h} b_{h}=\rho h_{i j} .
$$

From the above definition, we have

$$
L \dot{\partial}_{j} b_{i}=\rho h_{i j},
$$

which shows that $b_{i}$ is a function of directional argument also. H. Izumi [6] proved that the scalar $\rho$ is independent of directional argument. Gupta and Pandey [4] proved that if the $h$-vector $b_{i}$ is gradient then the scalar $\rho$ is constant. M. Matsumoto [9] discussed the Cartan connection of Randers change of Finsler metric, while B. N. Prasad [13] obtained the Cartan connection of $\left(M^{n},{ }^{*} L\right)$ where ${ }^{*} L(x, y)$ is given by ${ }^{*} L(x, y)=L(x, y)+b_{i}(x, y) y^{i}$, and $b_{i}(x, y)$ is an $h$-vector. Gupta and Pandey $[2,3]$ discussed the hypersurface of a Finsler space whose metric is given by certain transformation with an $h$-vector.

A geodesic of $F^{n}=\left(M^{n}, L\right)$ is given by system of differential equation

$$
\frac{d^{2} x^{i}}{d x^{2}}+2 G^{i}\left(x, \frac{d x}{d s}\right)=0,
$$

where $G^{i}(x, y)$ are positively homogeneous of degree two in $y^{i}$ and are given by

$$
2 G^{i}=g^{i j}\left(y^{r} \dot{\partial}_{j} \partial_{r} F-\partial_{j} F\right), \quad F=\frac{L^{2}}{2},
$$

where $g^{i j}$ is the inverse of $g_{i j}$.

A transformation from $F^{n}=\left(M^{n}, L\right)$ to $\bar{F}^{n}=\left(M^{n}, \bar{L}\right)$ is called projective change if a geodesic on $F^{n}$ is also a geodesic on $\bar{F}^{n}$ and vice-versa. The change $L \mapsto \bar{L}$ is projective change if and only if there exits a scaler function $P(x, y)$ which is positive homogeneous of degree one in $y^{i}$ and satisfies [1]

$$
\bar{G}^{i}(x, y)=G^{i}(x, y)+P(x, y) y^{i} .
$$

I. Y. Lee and H. S. Park [12] studied projective change between a Finsler space with $(\alpha, \beta)$-metric and associated Riemannian space. M. Hashiguchi and Y. Ichijyo [5] obtained the condition for Randers change to be projective whereas Gupta and Pandey[4] derived the condition for Kropina change to be projective. Many authors $[7,11,15]$ studied the projective change in different spaces.

In the present paper, we consider a Finsler space ${ }^{*} F^{n}=\left(M^{n},{ }^{*} L\right)$, whose metric function ${ }^{*} L$, an $h$-exponential change of metric, is given by

$$
{ }^{*} L=L e^{\frac{\beta}{L}},
$$

where $\beta=b_{i}(x, y) y^{i}$ and $b_{i}$ is an $h$-vector. And we obtain the relation between Cartan connection coefficients of $F^{n}$ and ${ }^{*} F^{n}$. We also derive the condition for an $h$-exponential change of metric to be projective. 


\section{2. $\quad$ Finsler space ${ }^{*} F^{n}=\left(M^{n},{ }^{*} L\right)$}

We shall use the following notations $L_{i}=\dot{\partial}_{i} L=l_{i}, \quad L_{i j}=\dot{\partial}_{i} \dot{\partial}_{j} L, \quad L_{i j k}=\dot{\partial}_{i} \dot{\partial}_{j} \dot{\partial}_{k} L$. The quantities corresponding to ${ }^{*} F^{n}$ is denoted by asterisk over that quantity.

From (1.6), we have

$$
\begin{gathered}
{ }^{*} L_{i}=e^{\tau}\left(m_{i}+l_{i}\right) . \\
{ }^{*} L_{i j}=e^{\tau}(1+\rho-\tau) L_{i j}+\frac{e^{\tau}}{L} m_{i} m_{j} . \\
{ }^{*} L_{i j k}=e^{\tau}(1+\rho-\tau) L_{i j k}+(\rho-\tau) \frac{e^{\tau}}{L}\left[m_{i} L_{j k}+m_{j} L_{i k}+m_{k} L_{i j}\right] \\
-\frac{e^{\tau}}{L^{2}}\left[m_{j} m_{k} l_{i}+m_{i} m_{k} l_{j}+m_{i} m_{j} l_{k}-m_{i} m_{j} m_{k}\right],
\end{gathered}
$$

where $\tau=\frac{\beta}{L}, m_{i}=b_{i}-\tau l_{i}$. The normalised supporting element, the metric tensor and Cartan tensor of ${ }^{*} F$ are obtained as

$$
{ }^{*} l_{i}=e^{\tau}\left(m_{i}+l_{i}\right)
$$

$$
{ }^{*} C_{i j k}=\nu e^{2 \tau} C_{i j k}+\frac{2}{L} e^{2 \tau} m_{i} m_{j} m_{k}+\frac{1}{2 L} e^{2 \tau}(2 \nu-1)\left(m_{i} h_{k j}+m_{j} h_{k i}+m_{k} h_{i j}\right)
$$

where $\nu=1+\rho-\tau$.

For the computation of the inverse metric tensor, we use the following lemma [8] :

Lemma 2.1. Let $\left(m_{i j}\right)$ be a non-singular matrix and $l_{i j}=m_{i j}+n_{i} n_{j}$. The elements $l^{i j}$ of the inverse matrix and determinant of the matrix $\left(l_{i j}\right)$ are given by

$$
l^{i j}=m^{i j}-\left(1+n_{k} n^{k}\right)^{-1} n^{i} n^{j}, \quad \operatorname{det}\left(l_{i j}\right)=\left(1+n_{k} n^{k}\right) \operatorname{det}\left(m_{i j}\right)
$$

respectively, where $m^{i j}$ are elements of inverse matrix $\left(m_{i j}\right)$ and $n^{k}=m^{k i} n_{i}$.

The inverse metric tensor of ${ }^{*} F^{n}$ is derived as follows :

$$
{ }^{*} g^{i j}=\frac{e^{-2 \tau}}{\nu}\left[g^{i j}-\frac{1}{m^{2}+\nu} b^{i} b^{j}+\frac{\tau-\nu}{m^{2}+\nu}\left(b^{i} l^{j}+b^{j} l^{i}\right)-l^{i} l^{j}\left\{\frac{\tau-\nu}{m^{2}+\nu}\left(m^{2}+\tau\right)-\rho\right\}\right],
$$


where $b$ is magnitude of the vector $b^{i}=g^{i j} b_{j}$.

From (2.6) and (2.7), we obtain

$$
\begin{aligned}
{ }^{*} C_{i j}^{h}= & C_{i j}^{h}+\frac{1}{m^{2}+\nu} C_{i j k} b^{k}\left(-b^{h}+2 \tau l^{h}-\rho l^{h}-l^{h}\right) \\
& +\frac{2}{\nu L}\left[m_{i} m_{j} m^{h}+\frac{1}{m^{2}+\nu} m_{i} m_{j} m^{2}\left(-b^{h}+2 \tau l^{h}-\rho l^{h}-l^{h}\right)\right] \\
& +\frac{1}{2 \nu L}(2 \nu-1)\left[m_{i} h_{j}^{h}+m_{j} h_{i}^{h}+m^{h} h_{i j}\right. \\
& \left.+\frac{1}{m^{2}+\nu}\left(-b^{h}+2 \tau l^{h}-\rho l^{h}-l^{h}\right)\left(2 m_{i} m_{j}+m^{2} h_{i j}\right)\right] .
\end{aligned}
$$

\section{Cartan connection of the space ${ }^{*} F^{n}$}

Let $C^{*} \Gamma=\left({ }^{*} F_{j k}^{i},{ }^{*} N_{j}^{i},{ }^{*} C_{j k}^{i}\right)$ be the Cartan connection for the Finsler space ${ }^{*} F^{n}=$ $\left(M^{n},{ }^{*} L\right)$. Since $L_{i \mid j}=0$ for the Cartan connection, we have

$$
\partial_{j} L_{i}=L_{r} F_{i j}^{r}+\dot{\partial}_{r} L_{i} N_{j}^{r}
$$

Differentiating (2.1) with respect to $x^{j}$, and using (1.1) and (3.1), we get

${ }^{*} L_{i r}{ }^{*} N_{j}^{r}+{ }^{*} L_{r}{ }^{*} F_{i j}^{r}=\left[e^{\tau} \nu L_{i r}+\frac{e^{\tau}}{L} m_{r} m_{i}\right] N_{j}^{r}+\left[e^{\tau}\left(m_{r}+l_{r}\right)\right] F_{i j}^{r}+\frac{e^{\tau} \beta_{j} m_{i}}{L}+e^{\tau} b_{i \mid j}$.

Equation (3.2) serves the purpose to find relation between a Cartan connection of ${ }^{*} F^{n}$ and $F^{n}$. For this, we put

$$
D_{j k}^{i}={ }^{*} F_{j k}^{i}-F_{j k}^{i}
$$

With the help of (3.3), the equation (3.2) becomes

$$
\left[e^{\tau} \nu L_{i r}+\frac{e^{\tau}}{L} m_{i} m_{r}\right] D_{0 j}^{r}+\left[e^{\tau}\left(m_{r}+l_{r}\right)\right] D_{i j}^{r}=\frac{e^{\tau} \beta_{\mid j} m_{i}}{L}+e^{\tau} b_{i \mid j}
$$

where the subscript ' 0 ' denote the contraction by $y^{i}$.

Differentiating (2.2) with respect to $x^{k}$, and using (1.1) and (3.1), we have

$$
\begin{aligned}
& e^{\tau} \nu\left[L_{i j r} D_{0 k}^{r}+L_{r j} D_{i k}^{r}+L_{i r} D_{j k}^{r}\right]+(\nu-1) \frac{e^{\tau}}{L}\left[m_{r} L_{i j}+m_{i} L_{j r}+m_{j} L_{i r}\right] D_{0 k}^{r} \\
& -\frac{e^{\tau}}{L^{2}}\left[m_{i} m_{j} l_{r}+m_{j} m_{r} l_{i}+m_{r} m_{i} l_{r}-m_{i} m_{j} m_{r}\right] D_{0 k}^{r}+\frac{e^{\tau}}{L}\left[m_{r} m_{j} D_{i k}^{r}+m_{i} m_{r} D_{j k}^{r}\right] \\
& -\frac{e^{\tau}(\nu-1)}{L} L_{i j} \beta_{\mid k}-\frac{e^{\tau}}{L^{2}} \beta_{\mid k} m_{i} m_{j}-e^{\tau} \rho_{k} L_{i j}=0,
\end{aligned}
$$

where $\rho_{k}=\rho_{\mid k}=\partial_{k} \rho$. 
Theorem 3.1. The Cartan connection of ${ }^{*} F^{n}$ is completely determined by the equations (3.4) and (3.5).

To prove this, first we propose the following lemma :

Lemma 3.1. The system of equations

$$
\begin{aligned}
& (i){ }^{*} L_{i r} A^{r}=B_{i} \\
& (\text { ii }) \quad{ }^{*} L_{r} A^{r}=B
\end{aligned}
$$

has a unique solution $A^{r}$ for given $B$ and $B_{i}$.

Proof. Using (2.2), equation ( $i$ ) becomes

$$
\frac{e^{\tau}}{L}\left[\nu\left(g_{i r}-l_{i} l_{r}\right)+m_{i} m_{r}\right] A^{r}=B_{i}
$$

Contracting by $b^{i}$, we get

$$
m_{r} A^{r}=\frac{L B_{\beta}}{e^{\tau}}\left(m^{2}+\nu\right)^{-1}
$$

here we used subscript $\beta$ to denote the contraction by $b^{i}$, i.e. $B_{\beta}=B_{i} b^{i}$. From (2.1) and (ii), we have

$$
l_{r} A_{r}=\frac{B}{e^{\tau}}-\frac{L B_{\beta}}{e^{\tau}}\left(m^{2}+\nu\right)^{-1} .
$$

Using (3.7) and (3.8), equation(3.6) becomes

$$
g_{i r} A^{r}=\frac{L B_{i}}{\nu e^{\tau}}+l_{i}\left[\frac{B}{e^{\tau}}-\frac{L B_{\beta}}{e^{\tau}}\left(m^{2}+\nu\right)^{-1}\right]-\frac{m_{i} L B_{\beta}}{\nu e^{\tau}}\left(m^{2}+\nu\right)^{-1},
$$

contracting by $g^{i j}$, we have

$$
A^{j}=\frac{L B^{j}}{\nu e^{\tau}}+l^{j}\left[\frac{B}{e^{\tau}}-\frac{L B_{\beta}}{e^{\tau}}\left(m^{2}+\nu\right)^{-1}\right]-\frac{m^{j} L B_{\beta}}{\nu e^{\tau}}\left(m^{2}+\nu\right)^{-1},
$$

which is concrete form of the solution $A^{j}$.

Now we are in the position to prove the theorem. We will find an explicit expression of difference tensor $D_{j k}^{i}$ in three steps. Firstly, we will find $D_{00}^{i}$ and then $D_{0 k}^{i}$ and in the last $D_{j k}^{i}$.

Taking symmetric and skew-symmetric part of (3.4), we have

$$
\begin{aligned}
2 e^{\tau}\left(m_{r}+l_{r}\right) D_{i j}^{r}+\left[\nu e^{\tau} L_{i r}+\frac{e^{\tau}}{L} m_{i} m_{r}\right] D_{0 j}^{r}+ & {\left[\nu e^{\tau} L_{j r}+\frac{e^{\tau}}{L} m_{j} m_{r}\right] D_{0 i}^{r} } \\
& =\frac{e^{\tau}}{L}\left(\beta_{\mid j} m_{i}+\beta_{\mid i} m_{j}\right)+2 e^{\tau} E_{i j},
\end{aligned}
$$


and

$$
\begin{aligned}
{\left[\nu e^{\tau} L_{i r}+\frac{e^{\tau}}{L} m_{i} m_{r}\right] D_{0 j}^{r}-} & {\left[\nu e^{\tau} L_{j r}+\frac{e^{\tau}}{L} m_{j} m_{r}\right] D_{0 i}^{r} } \\
& =\frac{e^{\tau}}{L}\left(\beta_{\mid j} m_{i}-\beta_{\mid i} m_{j}\right)+2 e^{\tau} F_{i j}
\end{aligned}
$$

where $2 E_{i j}=b_{j \mid i}+b_{i \mid j}, \quad 2 F_{i j}=b_{i \mid j}-b_{j \mid i}$.

Contracting (3.10) and (3.11) by $y^{j}$, we get

$$
2 e^{\tau}\left(m_{r}+l_{r}\right) D_{0 i}^{r}+\left[\nu e^{\tau} L_{i r}+\frac{e^{\tau}}{L} m_{i} m_{r}\right] D_{00}^{r}=\frac{e^{\tau}}{L} \beta_{\mid 0} m_{i}+2 e^{\tau} E_{i 0},
$$

and

$$
\left[\nu e^{\tau} L_{i r}+\frac{e^{\tau}}{L} m_{i} m_{r}\right] D_{00}^{r}=\frac{e^{\tau}}{L} \beta_{\mid 0} m_{i}+2 e^{\tau} F_{i 0},
$$

which may be re-written as

$$
{ }^{*} L_{i r} D_{00}^{r}=\frac{e^{\tau}}{L} \beta_{\mid 0} m_{i}+2 e^{\tau} F_{i 0},
$$

where $\beta_{\mid 0}=\beta_{\mid j} y^{j}$. Transvecting (3.13) by $m^{i}$, we obtain

$$
m_{r} D_{00}^{r}=\left(m^{2}+\nu\right)^{-1}\left(\beta_{\mid 0} m^{2}+2 L F_{\beta 0}\right) .
$$

Contracting (3.12) by $y^{i}$, we get

$$
2 e^{\tau}\left(m_{r}+l_{r}\right) D_{00}^{r}=2 e^{\tau} E_{00} .
$$

i.e.

$$
{ }^{*} L_{r} D_{00}^{r}=e^{\tau} E_{00} .
$$

Applying Lemma 3.1 in equation (3.14) and (3.16), we have

$$
\begin{aligned}
D_{00}^{i}= & \frac{L}{\nu e^{\tau}}\left[\frac{e^{\tau}}{L} \beta_{\mid 0} m^{i}+2 e^{\tau} F_{0}^{i}\right]+l^{i}\left[E_{00}-\frac{L}{e^{\tau}}\left(m^{2}+\nu\right)^{-1}\left(\frac{e^{\tau}}{L} \beta_{\mid 0} m^{2}+2 e^{\tau} F_{\beta 0}\right)\right] \\
& -\frac{m^{i} L}{\nu e^{\tau}}\left(m^{2}+\nu\right)^{-1}\left[\frac{e^{\tau}}{L} \beta_{\mid 0} m^{2}+2 e^{\tau} F_{\beta 0}\right] .
\end{aligned}
$$

Here we used $m^{i} b_{i}=m_{i} m^{i}=m^{2}$. Also we note that $E_{00}=E_{i j} y^{i} y^{j}=b_{i \mid j} y^{i} y^{j}=$ $\left(b_{i} y^{i}\right)_{\mid j} y^{j}=\beta_{\mid 0}, F_{0}^{i}=g^{i j} F_{j 0}$.

Secondly, applying Christoffel process with respect to indices $i, j, k$ in equation 
(3.5), we have

(3.18)

$$
\begin{aligned}
& \nu e^{\tau}\left[L_{i j r} D_{0 k}^{r}+L_{j k r} D_{0 i}^{r}-L_{k i r} D_{0 j}^{r}\right]+2 D_{i k}^{r}\left[\nu e^{\tau} L_{j r}+\frac{e^{\tau}}{L} m_{r} m_{j}\right] \\
& +\frac{e^{\tau}}{L} D_{0 k}^{r} \mathfrak{S}_{(r i j)}\left[(\nu-1) m_{r} L_{i j}-\frac{m_{i} m_{j} l_{r}}{L}\right]+\frac{e^{\tau}}{L} D_{0 i}^{r} \mathfrak{S}_{(r j k)}\left[(\nu-1) m_{r} L_{j k}-\frac{m_{j} m_{k} l_{r}}{L}\right] \\
& -\frac{e^{\tau}}{L} D_{0 j}^{r} \mathfrak{S}_{(r k i)}\left[(\nu-1) m_{r} L_{k i}-\frac{m_{k} m_{i} l_{r}}{L}\right]-e^{\tau}\left[\rho_{k} L_{i j}+\rho_{i} L_{j k}-\rho_{j} L_{k i}\right] \\
& -(\nu-1) \frac{e^{\tau}}{L}\left(\beta_{\mid k} L_{i j}+\beta_{\mid i} L_{j k}-\beta_{\mid j} L_{k i}\right)-\frac{e^{\tau}}{L^{2}}\left[\beta_{\mid k} m_{i} m_{j}+\beta_{\mid i} m_{j} m_{k}-\beta_{\mid j} m_{k} m_{i}\right] \\
& +\frac{e^{\tau}}{L^{2}}\left[m_{i} m_{j} m_{r} D_{0 k}^{r}+m_{j} m_{k} m_{r} D_{0 i}^{r}-m_{k} m_{i} m_{r} D_{0 j}^{r}\right]=0
\end{aligned}
$$

where $\mathfrak{S}_{(i j k)}$ denote cyclic interchange of indices $i, j, k$ and summation. Contracting by $y^{k}$, above equation becomes

$$
\begin{aligned}
& \nu e^{\tau}\left[L_{i j r} D_{00}^{r}-L_{j r} D_{0 i}^{r}+L_{i r} D_{0 j}^{r}\right]+2 D_{0 i}^{r}\left[\nu e^{\tau} L_{j r}+\frac{e^{\tau}}{L} m_{r} m_{j}\right] \\
& +\frac{e^{\tau}}{L} D_{00}^{r} \mathfrak{S}_{(r i j)}\left[(\nu-1) m_{r} L_{i j}-\frac{m_{i} m_{j} l_{r}}{L}\right]-\frac{e^{\tau}}{L} D_{0 i}^{r} \frac{m_{r} m_{j} l_{k}}{L} y^{k} \\
& +\frac{e^{\tau}}{L} D_{0 j}^{r} \frac{m_{r} m_{i} l_{k}}{L} y^{k}+\frac{e^{\tau}}{L^{2}} m_{i} m_{j} m_{r} D_{00}^{r}-(\nu-1) \frac{e^{\tau}}{L} \beta_{\mid 0} L_{i j} \\
& -\frac{e^{\tau}}{L^{2}} \beta_{\mid 0} m_{i} m_{j}-e^{\tau} \rho_{0} L_{i j}=0 .
\end{aligned}
$$

Adding (3.11) and (3.19), we have

$$
{ }^{*} L_{i r} D_{0 j}^{r}=G_{i j}
$$

where

$$
\begin{aligned}
2 G_{i j}= & \frac{e^{\tau}}{L}\left(\beta_{\mid j} m_{i}-\beta_{\mid i} m_{j}\right)-e^{\tau} \nu L_{i j r} D_{00}^{r}-\frac{e^{\tau}}{L} D_{00}^{r} \mathfrak{S}_{(r i j)}\left[(\nu-1) m_{r} L_{i j}-\frac{m_{i} m_{j} m_{r}}{L}\right] \\
& +2 e^{\tau} F_{i j}-\frac{e^{\tau}}{L^{2}} m_{r} m_{i} m_{j} D_{00}^{r}+\frac{(\nu-1)}{L} e^{\tau} \beta_{\mid 0} L_{i j}+\frac{e^{\tau}}{L^{2}} B_{0} m_{i} m_{j}+e^{\tau} \rho_{0} L_{i j} .
\end{aligned}
$$

Equation (3.12) can be written as

$$
{ }^{*} L_{r} D_{0 j}^{r}=G_{j}
$$

where

$$
2 G_{j}=\frac{e^{\tau}}{L} \beta_{\mid 0} m_{j}+2 e^{\tau} E_{j 0}+\left[-e^{\tau} \nu L_{j r}-\frac{e^{\tau} m_{j} m_{r}}{L}\right] D_{00}^{r} .
$$

Using (3.13), the above equation may be written as

$$
G_{j}=e^{\tau}\left(E_{j 0}-F_{j 0}\right) .
$$


Applying Lemma 3.1 in equation (3.20) and (3.22), we obtain

$$
D_{0 j}^{i}=\frac{L G_{j}^{i}}{\nu e^{\tau}}+\frac{l^{i}}{e^{\tau}}\left[G_{j}-L G_{\beta j}\left(m^{2}+\nu\right)^{-1}\right]-\frac{m^{i} L G_{\beta j}}{\nu e^{\tau}}\left(m^{2}+\nu\right)^{-1} .
$$

Finally, the equation (3.10) may be written as

where

$$
{ }^{*} L_{r} D_{i k}^{r}=H_{i k},
$$

$$
\begin{aligned}
2 H_{i k}=\frac{e^{\tau}}{L}\left(\beta_{\mid k} m_{i}+\beta_{\mid i} m_{k}\right) & +e^{\tau} E_{i k}-\left[e^{\tau} \nu L_{i r}+\frac{e^{\tau}}{L} m_{i} m_{r}\right] D_{0 k}^{r} \\
& -\left[e^{\tau} \nu L_{k r}+\frac{e^{\tau}}{L} m_{k} m_{r}\right] D_{0 i}^{r} .
\end{aligned}
$$

Equation (3.18) may be written as

where

$$
{ }^{*} L_{r j} D_{i k}^{r}=H_{j i k},
$$

$$
\begin{aligned}
& 2 H_{j i k}=-\nu e^{\tau}\left[L_{i j r} D_{0 k}^{r}+L_{j k r} D_{0 i}^{r}-L_{k i r} D_{0 j}^{r}\right]+e^{\tau}\left[\rho_{k} L_{i j}+\rho_{i} L_{j k}-\rho_{j} L_{k i}\right] \\
& -\frac{e^{\tau}}{L} D_{0 k}^{r} \mathfrak{S}_{(r i j)}\left[(\nu-1) m_{r} L_{i j}-\frac{m_{i} m_{j} l_{r}}{L}\right]-\frac{e^{\tau}}{L} D_{0 i}^{r} \mathfrak{S}_{(r j k)}\left[(\nu-1) m_{r} L_{j k}-\frac{m_{j} m_{k} l_{r}}{L}\right] \\
& +\frac{e^{\tau}}{L} D_{0 j}^{r} \mathfrak{S}_{(r k i)}\left[(\nu-1) m_{r} L_{k i}-\frac{m_{k} m_{i} l_{r}}{L}\right] \\
& +(\nu-1) \frac{e^{\tau}}{L}\left(\beta_{\mid k} L_{i j}+\beta_{\mid i} L_{j k}-\beta_{\mid j} L_{k i}\right)+\frac{e^{\tau}}{L^{2}}\left[\beta_{\mid k} m_{i} m_{j}+\beta_{\mid i} m_{j} m_{k}-\beta_{\mid j} m_{k} m_{i}\right] \\
& -\frac{e^{\tau}}{L^{2}}\left[m_{i} m_{j} m_{r} D_{0 k}^{r}+m_{j} m_{k} m_{r} D_{0 i}^{r}-m_{k} m_{i} m_{r} D_{0 j}^{r}\right] .
\end{aligned}
$$

Applying Lemma 3.1 in (3.25) and (3.27), we have

$$
D_{i k}^{j}=\frac{L H_{i k}^{j}}{\nu e^{\tau}}+\frac{l^{j}}{e^{\tau}}\left[H_{i k}-L H_{\beta i k}\left(m^{2}+\nu\right)^{-1}\right]-\frac{m^{j} L}{\nu e^{\tau}} H_{\beta i k}\left(m^{2}+\nu\right)^{-1},
$$

where we put $H_{i k}^{j}=g^{j m} H_{m i k}$.

Thus in view of $(3.3)$, we get the Cartan connection coefficient ${ }^{*} F_{j k}^{i}$. This completes the proof of theorem (3.1).

Now, suppose Cartan connection coefficients for both spaces $F^{n}$ and ${ }^{*} F^{n}$ are same, i.e. ${ }^{*} F_{j k}^{i}=F_{j k}^{i}$. Then $D_{j k}^{i}=0$. But then equations (3.12) and (3.13) implies that $E_{i 0}=F_{i 0}$, and hence

$$
b_{0 \mid i}=0,
$$

i.e. $\beta_{\mid i}=0$. Differentiating $\beta_{\mid i}=0$ partially with respect to $y^{j}$ and applying commutation formulae $\dot{\partial}_{j}\left(\beta_{\mid i}\right)-\left(\dot{\partial}_{j} \beta\right)_{\mid i}=-\left(\dot{\partial}_{r} \beta\right) C_{i j \mid 0}^{r}$, we get

$$
b_{j \mid i}=b_{r} C_{i j \mid 0}^{r} .
$$


From the above equation, we conclude that $F_{i j}=0$. M. K. Gupta and P. N. Pandey [4] has proved that if $h$-vector $b_{i}$ is gradient, i.e. $F_{i j}=0$ then $\rho$ is constant, i.e. $\rho_{i}=\rho_{\mid i}=0$. Taking $h$-covariant derivative of $L C_{i j}^{r} b_{r}=\rho h_{i j}$ and using $L_{\mid k}=0, \rho_{\mid k}=0$ and $h_{i j \mid k}=0$, we have

$$
\left(b_{r} C_{i j}^{r}\right)_{k}=\frac{\rho}{L} h_{i j}=0,
$$

i.e.,

$$
b_{r \mid k} C_{i j}^{r}+b_{r} C_{i j \mid k}^{r}=0 .
$$

From (3.31), $b_{r \mid k}=b_{k \mid r}$ and hence above equation becomes

$$
b_{k \mid r} C_{i j}^{r}+b_{r} C_{i j \mid k}^{r}=0 .
$$

Transvecting by $y^{k}$, we have $b_{0 \mid r} C_{i j}^{r}+b_{r} C_{i j \mid 0}^{r}=0$. Using (3.30) and (3.31), we conclude that $b_{i \mid j}=0$.

Conversely, $b_{i \mid j}=0$ implies that $E_{i j}=0=F_{i j}$ and $\beta_{\mid i}=\beta_{\mid i}=b_{j \mid i}=0 . F_{i j}=0$ implies that $\rho_{i}=\rho_{\mid i}=0[4]$. Therefore from (3.17), we get $D_{00}^{i}=0$ and then $G_{i j}=0$ and $G_{j}=0$. This gives $D_{0 j}^{i}=0$ and then $H_{j i k}=0$ and $H_{i k}=0$. Therefore (3.29) implies that $D_{j k}^{i}=0$. Thus, we have:

Theorem 3.2. For an h-exponential change of metric, the Cartan connection coefficients for both spaces $F^{n}$ and ${ }^{*} F^{n}$ are same if and only if the h-vector $b_{i}$ is parallel with respect to Cartan connection of $F^{n}$.

Now transvecting (3.3) by $y^{j}$ and using $F_{j k}^{i} y^{j}=G_{k}^{i}$, we obtain

$$
{ }^{*} G_{k}^{i}=G_{k}^{i}+D_{0 k}^{i} .
$$

Transvecting again the above equation by $y^{k}$ and using $G_{k}^{i} y^{k}=2 G^{i}$, we get

$$
2{ }^{*} G^{i}=2 G^{i}+D_{00}^{i}
$$

Differentiating (3.32) partially with respect to $y^{h}$ and using $\dot{\partial}_{h} G_{k}^{i}=G_{k h}^{i}$, we have

$$
{ }^{*} G_{k h}^{i}=G_{k h}^{i}+\dot{\partial}_{h} D_{0 k}^{i},
$$

where $G_{k h}^{i}$ are Berwald connection coefficients.

Now, if the $h$-vector $b_{i}$ is parallel with respect to Cartan connection of $F^{n}$ then by Theorem (3.2), the Cartan connection coefficients for both spaces $F^{n}$ and ${ }^{*} F^{n}$ are same, therefore $D_{j k}^{i}=0$. Hence from (3.34), we get ${ }^{*} G_{k h}^{i}=G_{k h}^{i}$.

Thus, we have:

Theorem 3.3. For an h-exponential change of metric, if an h-vector $b_{i}$ is parallel with respect to Cartan connection of $F^{n}$ then Barwald connection coefficients for both spaces $F^{n}$ and ${ }^{*} F^{n}$ are the same. 


\section{Condition for h-exponential change of metric to be projective}

Now, we find the condition for exponential change with $h$-vector to be projective. From (3.33), it follows that exponential change with $h$-vector to be projective if and only if $D_{00}^{i}=2 P y^{i}$. Then from (3.17), we get

$$
\begin{aligned}
2 P y^{i}= & \frac{L}{\nu e^{\tau}}\left[\frac{e^{\tau}}{L} \beta_{\mid 0} m^{i}+2 e^{\tau} F_{0}^{i}\right]+l^{i}\left[E_{00}-\frac{L}{e^{\tau}}\left(m^{2}+\nu\right)^{-1}\left(\frac{e^{\tau}}{L} \beta_{\mid 0} m^{2}+2 e^{\tau} F_{\beta 0}\right)\right] \\
& -\frac{m^{i} L}{\nu e^{\tau}}\left(m^{2}+\nu\right)^{-1}\left[\frac{e^{\tau}}{L} \beta_{\mid 0} m^{2}+2 e^{\tau} F_{\beta 0}\right] .
\end{aligned}
$$

Transvecting (4.1) by $y_{i}$ and using $m^{i} y_{i}=0, F_{0}^{i} y_{i}=0$, we get

$$
P=\frac{y_{i} l^{i}}{2 L^{2}}\left[E_{00}-\frac{L}{e^{\tau}}\left(m^{2}+\nu\right)^{-1}\left(\frac{e^{\tau}}{L} \beta_{\mid 0} m^{2}+2 e^{\tau} F_{\beta 0}\right)\right] .
$$

Substituting the value of $P$ in(4.1), we get

$$
F_{0}^{i}=\frac{m^{i}}{2 L}\left(m^{2}+\nu\right)^{-1}\left(\beta_{\mid 0} m^{2}+2 L F_{\beta 0}\right)-\frac{\beta_{\mid 0} m^{i}}{2 L} .
$$

Using (3.15) in the above equation, we have

$$
F_{0}^{i}=\frac{m^{i}}{2 L} m_{r} D_{00}^{r}-\frac{\beta_{\mid 0} m^{i}}{2 L} .
$$

Transvecting by $g_{i j}$ to above equation, we have

$$
F_{i 0}=\frac{m_{i}}{2 L} m_{r} D_{00}^{r}-\frac{\beta_{\mid 0} m_{i}}{2 L} .
$$

Using (4.5) in (3.13) and referring $\nu \neq 0$, we obtain $L_{i r} D_{00}^{r}=0$, which transvecting by $m^{i}$ and using $L_{i r} m^{i}=\frac{1}{L} m_{r}$, we get $m_{r} D_{00}^{r}=0$, and then (4.5) becomes

$$
F_{i 0}=-\frac{\beta_{\mid 0} m_{i}}{2 L} .
$$

The equation (4.6) is necessary condition for $h$-exponential change to be projective change.

Conversely, if (4.6) satisfied, the equation (3.13) yields

$$
\left[e^{\tau} \nu L_{i r}+\frac{e^{\tau}}{L} m_{i} m_{r}\right] D_{00}^{r}=0 .
$$

Transvecting by $m^{i}$ and referring $\left(m^{2}+\nu\right) \neq 0$, we get $m_{r} D_{00}^{r}=0$ and then (3.17) gives $D_{00}^{i}=E_{00} l^{i}$. Therefore ${ }^{*} F^{n}$ is projective to $F^{n}$. Thus, we have:

Theorem 4.1. The h-exponential change given by (1.6) is projective if and only if condition (4.6) is satisfied. 


\section{R E F E R E N C E S}

[1] P. L. Antonelli, R.S. Ingarden and M. Matsumoto:The Theory of Sprays and Finsler Spaces with Applications in Physics and Biology, volume 58 of Fundamental Theories of Physics. Kluwer Academic Publishers Group, Dordrecht, 1993.

[2] M. K. Gupta and P. N. Pandey: On hyperspaces of a Finsler space with a special metric. Acta Math. Hunger., 120(1-2),(2008), 165-177.

[3] M. K. Gupta and P. N. Pandey: Hyperspaces of conformally and h-conformally related Finsler spaces. Acta Math. Hunger., 123(3),(2009), 257-264.

[4] M. K. Gupta and P. N. Pandey: Finsler space subjected to a Kropina change with an h-vector. Facta Universitatis (NIŠ) Ser. Math. Inform., 30(4),(2015), 513-525.

[5] M. Hashiguchi and Y. Ichijyo: Randers space with rectilinear geodesics.Rep. Fac. Sci. Kagoshima Univ., (Math., Phys. and Chem.), 13,(1980), 33-40.

[6] H. Izumi: Conformal transformation of Finsler spaces II. An h-conformally flat Finsler space.Tensor N.S. 34,(1980), 337-359.

[7] M. Kitayama and T. Tani: Hypersurfaces of projective $\beta$-changes. Memoirs of the Scientific Sections of the Romanian Academy Tome XXXIV, , 2011, 55-63.

[8] M. Matsumoto: On C-reducible Finsler spaces. Tensor N.S., (24), 1972, 29-37.

[9] M. Matsumoto: On Finsler space with Rander's metric and special forms of important tensors. J.Math. Kyoto Univ., 14(3),(1974), 477-498.

[10] M. Matsumoto: Foundation of Finsler geometry and special Finsler spaces. Kaiseigha press, Saikawa, Otsu, Japan, 1986.

[11] N. L. Youssef, S. H. Abed and A. Soleiman: Intrinsic theory of projective change in finsler geometry.Rend. Circ. Mat. Palermo, 60, (2011), 263281.

[12] I. Y. Lee and H. S. Park: Projective changes between a Finsler space with $(\alpha, \beta)$-metric and the associated Riemannian metric. Canad. J. Math., 60, 2008, 443-456.

[13] B. N. Prasad: On the torsion tensors $R_{h j k}$ and $P_{h j k}$ of Finsler spaces with a metric $d s=\left(g_{i} j(d x) d x^{i} d x^{j}\right)^{\frac{1}{2}}+b_{i}(x, y) d x^{i}$. Indian J. pure appl. math, 21(1), 1990, 27-39.

[14] H. Rund: The differential geometry of Finsler spaces. Springer-Verlog, 1959.

[15] H. S. Shukla, B. N. Prasad and O. P. Pandey: Exponential Change of Finsler metric. Int. J. Contemp. Math. Sciences, 7, (2012), 2253-2263.

Manish Kumar Gupta

Department of Pure \& Applied Mathematics

Guru Ghasudas Vishwavidyalaya

Bilaspur (C.G.), India

mkgiaps@gmail.com

Anil K. Gupta

Department of Pure \& Applied Mathematics

Guru Ghasudas Vishwavidyalaya

Bilaspur (C.G.), India

gupta. anil409@gmail.com 